\title{
Identification of novel long non-coding RNAs in triple-negative breast cancer
}

\author{
Xiaokun Shen ${ }^{1,2, *}$, Bojian Xie ${ }^{1, *}$, Zhaosheng Ma ${ }^{1, *}$, Wenjie Yu ${ }^{1}$, Wenmin Wang ${ }^{1}$, \\ Dong $\mathrm{Xu}^{1}{ }^{1}$ Xinqiang Yan ${ }^{1}$, Beibei Chen ${ }^{3}$, Longyao $\mathrm{Yu}^{4}{ }^{4}$ Jicheng $\mathrm{Li}^{2}$, Xiaobing Chen ${ }^{3}$, \\ Kan Ding ${ }^{4}$ and Feilin $\mathrm{Cao}^{1}$ \\ ${ }^{1}$ Department of Surgical Oncology, Taizhou Hospital, Wenzhou Medical University, Taizhou, Zhejiang, China \\ 2 Institute of Cell Biology, Zhejiang University, Hangzhou, China \\ 3 Department of Internal Oncology, Hennan Cancer Hospital, The Affiliated Cancer Hospital of Zhengzhou University, \\ Zhengzhou, Hennan, China \\ ${ }^{4}$ Glycobiology and Glycochemistry Lab, Shanghai Institute of Materia Medica, Chinese Academy of Sciences, Shanghai, \\ China \\ * These authors have contributed equally to this work \\ Correspondence to: Xiaobing Chen, email: 2290773710@qq.com
}

Kan Ding, email: dingkan@simm.ac.cn

Feilin Cao, email: tzyyrjk@hotmail.com

Keywords: triple-negative breast carcinomas; long non-coding RNAs

Received: January 06, $2015 \quad$ Accepted: May 02, $2015 \quad$ Published: June 10, 2015

This is an open-access article distributed under the terms of the Creative Commons Attribution License, which permits unrestricted use, distribution, and reproduction in any medium, provided the original author and source are credited.

\section{ABSTRACT}

Triple-negative breast carcinomas (TNBC) are characterized by particularly poor outcomes, and there are no established markers significantly associated with prognosis. Long non-coding RNAs (IncRNAs) are subclass of noncoding RNAs that have been recently shown to play critical roles in cancer biology. However, little is known about their mechanistic role in TNBC pathogenesis. In this report, we investigated the expression patterns of IncRNAs from TNBC tissues and matched normal tissues with Agilent Human IncRNA array. We identified 1,758 IncRNAs and 1,254 mRNAs that were differentially expressed ( $\geq 2$-fold change), indicating that many IncRNAs are significantly upregulated or downregulated in TNBC. Among these, XR_250621.1 and NONHSAT125629 were the most upregulated and downregulated IncRNAs respectively. qRT-PCR was employed to validate the microarray analysis findings, and results were consistent with the data from the microarrays. GO and KEGG pathway analysis were applied to explore the potential IncRNAs functions, some pathways including microtubule motor activity and DNA replication were identified in TNBC pathogenesis. Our study revealed that a set of IncRNAs were differentially expressed in TNBC tissues, suggesting that they may play role in TNBC. These results shed light on IncRNAs' biological functions and provide useful information for exploring potential therapeutic targets for breast cancer.

\section{INTRODUCTION}

Breast cancer is a heterogeneous neoplasm that comprises subtypes with substantial differences in biology and diverse clinical outcomes. As more molecularly targeted therapeutic agents are launched, more clinical remission problems are arising [1]. Therefore, identification of novel therapeutic targets is essential to combat breast cancers, especially those lacking estrogen receptor/progesterone receptor and ErbB2 receptor (triple negative breast cancer, TNBC). TNBC accounts for approximately $10-25 \%$ of all breast cancers and is of particular clinical interest due to its tendency to affect younger women and refractory to currently available targeted therapy. The molecular mechanisms for aggressive clinical behavior of TNBC are not fully 
Table 1: Top 30 aberrantly expressed IncRNAs in microarray for three pairs of TNBC and adjacent non-tumor tissues

\begin{tabular}{|c|c|c|c|c|c|c|c|c|c|c|c|}
\hline Target ID & FC (abs) & $\mathbf{p}$ & Regulation & C1 & $\mathrm{C2}$ & $\mathbf{C 3}$ & N1 & N2 & N3 & ncRNA_SeqID & Chr \\
\hline XR_250621.1 & 291.27 & 0.03 & down & 9.31 & 1.54 & 3.97 & 14.14 & 10.96 & 14.27 & humanseq 85285 & \\
\hline NONHSAT012762 & 164.35 & 0.04 & down & 8.00 & 1.56 & 1.72 & 12.20 & 8.81 & 12.35 & humanseq57970 & $\operatorname{chr} 10$ \\
\hline TCONS_12_00002973 & 144.14 & 0.04 & down & 8.09 & 1.33 & 2.98 & 12.59 & 8.67 & 12.65 & humanseq9097 & $\operatorname{chr} 10$ \\
\hline NONHSAG005629 & 135.22 & 0.04 & down & 8.22 & 1.49 & 3.54 & 12.67 & 9.20 & 12.62 & humanseq57206 & $\operatorname{chr} 10$ \\
\hline NONHSAG050621 & 133.56 & 0.01 & down & 4.09 & 1.29 & 2.81 & 8.88 & 8.32 & 12.17 & humanseq52435 & chr8 \\
\hline NONHSAT012761 & 128.01 & 0.04 & down & 7.94 & 1.24 & 3.46 & 12.45 & 8.49 & 12.70 & humanseq57969 & $\operatorname{chr} 10$ \\
\hline NONHSAT 127452 & 113.99 & 0.01 & down & 2.81 & 1.66 & 1.40 & 7.76 & 7.49 & 11.12 & humanseq52434 & chr8 \\
\hline XR_252733.1 & 105.54 & 0.03 & down & 7.33 & 1.50 & 2.51 & 11.57 & 8.26 & 11.68 & humanseq83671 & \\
\hline TCONS_12_00002976 & 99.20 & 0.04 & down & 8.08 & 1.55 & 3.87 & 12.28 & 8.59 & 12.53 & humanseq9047 & $\operatorname{chr} 10$ \\
\hline NONHSAT012773 & 90.54 & 0.04 & down & 6.95 & 1.74 & 1.87 & 11.13 & 7.47 & 11.46 & humanseq57973 & $\operatorname{chr} 10$ \\
\hline TCONS_12_00002971 & 62.00 & 0.04 & down & 7.64 & 3.46 & 2.77 & 11.67 & 8.00 & 12.06 & humanseq9095 & $\operatorname{chr} 10$ \\
\hline NONHSAT 121750 & 57.36 & 0.00 & down & 2.38 & 1.45 & 1.53 & 7.62 & 6.19 & 9.09 & humanseq49910 & $\operatorname{chr} 7$ \\
\hline NR_104061.1 & 55.50 & 0.04 & down & 7.57 & 3.66 & 2.70 & 11.53 & 8.19 & 11.59 & humanseq 86747 & 10 \\
\hline NONHSAT012774 & 54.24 & 0.03 & down & 6.95 & 4.22 & 2.64 & 11.26 & 8.11 & 11.72 & humanseq57974 & $\operatorname{chr} 10$ \\
\hline TCONS_12_00002977 & 52.98 & 0.04 & down & 5.64 & 1.44 & 1.34 & 9.75 & 5.82 & 10.02 & humanseq9048 & $\operatorname{chr} 10$ \\
\hline TCONS_12_00002974 & 52.00 & 0.04 & down & 7.59 & 4.23 & 2.76 & 11.56 & 8.29 & 11.83 & humanseq9098 & $\operatorname{chr} 10$ \\
\hline TCONS_12_00002972 & 41.39 & 0.04 & down & 6.35 & 2.56 & 2.81 & 10.39 & 6.79 & 10.65 & humanseq9096 & $\operatorname{chr} 10$ \\
\hline NR_026916.1 & 35.88 & 0.04 & down & 6.91 & 2.50 & 4.67 & 11.04 & 7.57 & 10.97 & humanseq 88505 & \\
\hline TCONS_12_00002970 & 35.17 & 0.04 & down & 5.42 & 1.80 & 4.24 & 9.97 & 6.42 & 10.49 & humanseq9094 & $\operatorname{chr} 10$ \\
\hline NONHSAT016222 & 33.04 & 0.03 & down & 5.97 & 2.48 & 3.51 & 10.08 & 6.89 & 10.12 & humanseq58945 & $\operatorname{chr} 10$ \\
\hline NONHSAT 136770 & 31.09 & 0.02 & down & 3.00 & 2.76 & 1.23 & 7.78 & 5.03 & 9.07 & humanseq55779 & $\operatorname{chrX}$ \\
\hline NONHSAT004026 & 27.10 & 0.02 & down & 5.15 & 2.65 & 1.85 & 8.86 & 6.53 & 8.55 & humanseq30344 & chrl \\
\hline NONHSAG048085 & 26.13 & 0.01 & down & 6.41 & 5.58 & 5.77 & 10.52 & 9.15 & 12.22 & humanseq49906 & $\operatorname{chr} 7$ \\
\hline NONHSAT009093 & 24.41 & 0.02 & down & 4.69 & 6.61 & 4.89 & 11.01 & 8.08 & 10.93 & humanseq32309 & chr1 \\
\hline NONHSAT125629 & 23.95 & 0.03 & up & 9.95 & 6.97 & 7.45 & 2.17 & 5.68 & 2.77 & humanseq51739 & chr8 \\
\hline NONHSAT066780 & 23.54 & 0.02 & down & 4.72 & 4.32 & 5.21 & 11.14 & 7.19 & 9.59 & humanseq77727 & $\operatorname{chr} 19$ \\
\hline NONHSAT012776 & 23.09 & 0.01 & down & 1.86 & 1.35 & 2.54 & 7.45 & 4.67 & 7.21 & humanseq57975 & $\operatorname{chr} 10$ \\
\hline XR_133419.2 & 22.26 & 0.01 & down & 1.35 & 2.01 & 1.30 & 6.48 & 4.06 & 7.56 & humanseq 84668 & \\
\hline NONHSAT 121746 & 21.61 & 0.03 & down & 1.30 & 3.18 & 2.19 & 6.98 & 4.35 & 8.64 & humanseq49907 & chr7 \\
\hline NONHSAT098133 & 20.15 & 0.00 & down & 1.46 & 1.46 & 1.35 & 6.44 & 4.45 & 6.37 & humanseq41971 & chr4 \\
\hline
\end{tabular}

understood. Various studies show that TNBC have broad and diverse categories for which additional subclasses are needed. Thus, there is considerable interest in understanding potential biomarkers that are significantly associated with TNBC prognosis.

Long non-coding RNAs (lncRNAs) are a subclass of noncoding RNAs (ncRNAs) and have sequence lengths of $200 \mathrm{bp}$ and above $[2,3]$. It has become increasingly apparent that lncRNAs contribute to tumor development through many different cellular processes, ranging from transcriptional and post-transcriptional regulation to the control of cell cycle distribution, cell differentiation and epigenetic modifications[4, 5]. LncRNAs modulate gene transcription regulation by rearranging chromatin via chromosomal looping and by affecting the binding of transcription factors. LncRNAs also affect miRNA functions by controlling pre-mRNA splicing or as miRNA sponges. Recently, accumulating evidence indicates that there is aberrant expression of lncRNAs in many cancer types, including glioma, lung, colorectal and hepatocellular cancers, etc [6-8]. Although prognostic lncRNAs expression signatures have been defined for some invasive breast carcinomas, little is known about
IncRNAs expression in TNBC, and the underlying pathways regulating TNBC aggressiveness remain poorly understood [9].

Here, we analyzed the expression patterns of lncRNAs and mRNAs in TNBC samples and compared them with the corresponding patterns in adjacent nontumorous tissue samples. We identified more than 1,200 unique lncRNAs and mRNAs significantly differentially expressed using microarray technology. Several of the differentially expressed IncRNAs were verified by qPCR in other 12 pairs of tissue samples. To determine the biological roles of these differentially expressed lncRNAs and mRNAs, GO and Pathway analyses were used. Coding-non-coding gene co-expression network identified many lncRNAs, such as lncRNA XR_250621.1, that potentially play a key role in TNBC pathogenesis. Our results suggest that lncRNAs expression patterns may provide new molecular biomarkers for the diagnosis of TNBC. 
Table 2. Top 30 aberrantly expressed mRNAs in microarray for three pairs of TNBC and adjacent non-tumor tissues

\begin{tabular}{|c|c|c|c|c|c|c|c|c|c|c|c|c|}
\hline Probe Name & Target ID & $\begin{array}{c}\text { Genbank } \\
\text { Accession }\end{array}$ & $\begin{array}{c}\text { FC } \\
(\mathrm{abs})\end{array}$ & $\mathbf{p}$ & Regulation & C1 & C2 & $\mathrm{C3}$ & N1 & N2 & N3 & Chr \\
\hline A_33_P3368985 & ANKRD30A & NM_052997 & 155.29 & 0.03 & down & 8.00 & 1.60 & 3.85 & 12.90 & 9.32 & 13.06 & chr10 \\
\hline A_23_P8820 & FABP4 & NM_001442 & 97.90 & 0.01 & down & 2.76 & 1.39 & 2.12 & 7.61 & 7.36 & 11.14 & chr8 \\
\hline A_23_P161940 & SCGB2A2 & NM_002411 & 92.77 & 0.03 & down & 2.51 & 1.48 & 2.36 & 11.72 & 4.99 & 9.24 & $\operatorname{chr} 11$ \\
\hline A_23_P218047 & KRT5 & NM_000424 & 63.47 & 0.03 & down & 9.25 & 3.97 & 5.83 & 12.93 & 10.68 & 13.41 & $\operatorname{chr} 12$ \\
\hline A_23_P12533 & ANKRD30A & NM_052997 & 60.17 & 0.04 & down & 6.66 & 1.79 & 2.69 & 10.86 & 7.00 & 11.01 & chr10 \\
\hline A_33_P3320683 & & & 51.36 & 0.03 & down & 7.43 & 2.81 & 4.11 & 11.59 & 8.11 & 11.70 & $\operatorname{chr} 10$ \\
\hline A_21_P0010304 & ANKRD30A & NM_052997 & 43.24 & 0.04 & down & 6.61 & 1.50 & 3.86 & 10.56 & 7.24 & 10.47 & $\operatorname{chr} 10$ \\
\hline A_23_P111583 & CD36 & NM_001001547 & 28.94 & 0.01 & down & 6.49 & 5.52 & 5.98 & 10.87 & 9.35 & 12.33 & $\operatorname{chr} 7$ \\
\hline A_23_P127781 & SCGB1D1 & NM_006552 & 28.85 & 0.03 & down & 1.81 & 2.28 & 2.15 & 9.00 & 4.21 & 7.57 & $\operatorname{chr} 11$ \\
\hline A_24_P273756 & ТP63 & NM_003722 & 27.89 & 0.05 & down & 6.25 & 1.86 & 3.92 & 10.11 & 6.49 & 9.83 & $\operatorname{chr} 3$ \\
\hline A_23_P206920 & MYH11 & NM_001040114 & 27.31 & 0.04 & down & 7.89 & 3.40 & 7.85 & 11.87 & 9.87 & 11.72 & chr16 \\
\hline A_24_P70183 & MYH11 & NM_001040113 & 26.71 & 0.05 & down & 7.85 & 3.31 & 7.82 & 11.80 & 9.61 & 11.78 & $\operatorname{chr} 16$ \\
\hline A_24_P260101 & MME & NM_007289 & 25.46 & 0.02 & down & 4.02 & 3.17 & 1.37 & 8.30 & 5.44 & 8.83 & chr3 \\
\hline A_33_P3319486 & & & 24.18 & 0.01 & down & 3.11 & 2.40 & 1.67 & 7.13 & 5.48 & 8.35 & $\operatorname{chr} 7$ \\
\hline A_24_P123408 & ABLIM3 & NM_014945 & 24.12 & 0.03 & down & 6.01 & 2.24 & 4.15 & 9.43 & 6.99 & 9.75 & $\operatorname{chr} 5$ \\
\hline A_23_P385861 & CDCA2 & NM_152562 & 22.83 & 0.03 & up & 9.83 & 6.92 & 7.36 & 2.58 & 5.42 & 2.57 & $\operatorname{chr} 8$ \\
\hline A_23_P323751 & FAM83D & NM_030919 & 22.31 & 0.03 & up & 8.36 & 5.87 & 7.33 & 1.44 & 5.10 & 1.59 & chr20 \\
\hline A_23_P356684 & ANLN & NM_018685 & 21.49 & 0.02 & up & 8.91 & 7.34 & 7.56 & 2.05 & 5.78 & 2.70 & $\operatorname{chr} 7$ \\
\hline A_24_P305050 & CD300LG & NM_145273 & 20.28 & 0.01 & down & 3.15 & 4.37 & 2.88 & 8.26 & 6.14 & 9.03 & $\operatorname{chr} 17$ \\
\hline A_23_P403284 & OTX1 & NM_014562 & 19.52 & 0.00 & up & 8.08 & 8.74 & 9.78 & 3.96 & 5.42 & 4.35 & chr2 \\
\hline A_23_P45185 & FIGF & NM_004469 & 19.18 & 0.02 & down & 1.99 & 2.55 & 1.51 & 6.84 & 4.25 & 7.75 & $\operatorname{chr} \mathrm{X}$ \\
\hline A_23_P77493 & TUBB3 & NM_006086 & 18.79 & 0.03 & up & 10.62 & 8.63 & 6.45 & 4.57 & 5.16 & 3.27 & chr16 \\
\hline A_23_P169437 & LCN2 & NM_005564 & 18.32 & 0.01 & up & 8.88 & 9.44 & 9.87 & 6.25 & 3.62 & 5.74 & chr9 \\
\hline A_24_P413884 & CENPA & NM_001809 & 17.22 & 0.02 & up & 7.19 & 6.01 & 6.33 & 1.32 & 4.51 & 1.38 & $\operatorname{chr} 2$ \\
\hline A_23_P315364 & CXCL2 & NM_002089 & 16.18 & 0.03 & down & 4.47 & 3.95 & 2.05 & 8.93 & 5.52 & 8.07 & chr4 \\
\hline A_23_P94422 & MELK & NM_014791 & 16.16 & 0.04 & up & 9.81 & 8.94 & 9.20 & 3.86 & 7.84 & 4.20 & $\operatorname{chr} 9$ \\
\hline A_24_P844984 & PIGR & NM_002644 & 16.09 & 0.02 & down & 4.57 & 6.24 & 4.60 & 10.07 & 7.47 & 9.90 & chr 1 \\
\hline A_23_P218369 & CCL14 & NM_032963 & 16.09 & 0.01 & down & 5.07 & 2.38 & 4.81 & 7.36 & 8.10 & 8.82 & $\operatorname{chr} 17$ \\
\hline A_24_P331150 & CYP4F22 & NM_173483 & 16.00 & 0.05 & down & 2.84 & 2.41 & 2.39 & 6.84 & 3.94 & 8.87 & $\operatorname{chr} 19$ \\
\hline A_23_P81280 & BTNL9 & NM_152547 & 15.91 & 0.02 & down & 3.23 & 3.72 & 4.91 & 7.64 & 6.66 & 9.54 & chr5 \\
\hline
\end{tabular}

\section{RESULTS}

\section{LncRNAs and mRNAs expression profiles in TNBC}

LncRNAs profiling detected 1,403 lncRNAs with significant differential expression levels with at least a two-fold change in TNBC tissues compared with paired normal tissues, with 853 up-regulated and 550 down-regulated respectively. The list of the top 30 differentially expressed lncRNAs identified by microarray analysis was shown in Table 1. Among the dysregulated lncRNAs transcripts, XR_250621.1 (humanseq85285) was the most down-regulated, with an FC of 291.3, whereas NONHSAT125629 (humanseq51739) was the most up-regulated, with an FC of 23.9. Using the same criteria as the lncRNAs, we found 574 up-regulated and 445 down-regulated mRNA transcripts. The most upregulated and downregulated mRNA transcripts were CDCA2 (NM_152562) and ANKRD30A (NM_052997), with FCs of 22.8 and 155.3, respectively (shown in Table
2). Hierarchical clustering of the lncRNAs and mRNAs profile was performed using cluster 3.0.2; Hierarchical clustering of the expression of the 1,403 lncRNAs and 1,019 mRNAs based on centered Pearson correlation clearly separated TNBC from normal tissues (Figure 1).

\section{Validation of the microarray data using qPCR}

The most upregulated lncRNA XR 250621.1 and downregulated lncRNA NONHSAT125629 were selected for validation using qPCR. In addition, two lncRNAs (ENST00000503938 and NONHSAT012762) were randomly selected to validate the microarray consistency using qPCR. The results demonstrated that lncRNAs NONHSAT125629 and ENST00000503938 were upregulated and that XR_250621.1 and NONHSAT012762 were down-regulated in the tumor samples compared with NT samples (Figure 2). These qPCR results are consistent with the microarray data. 


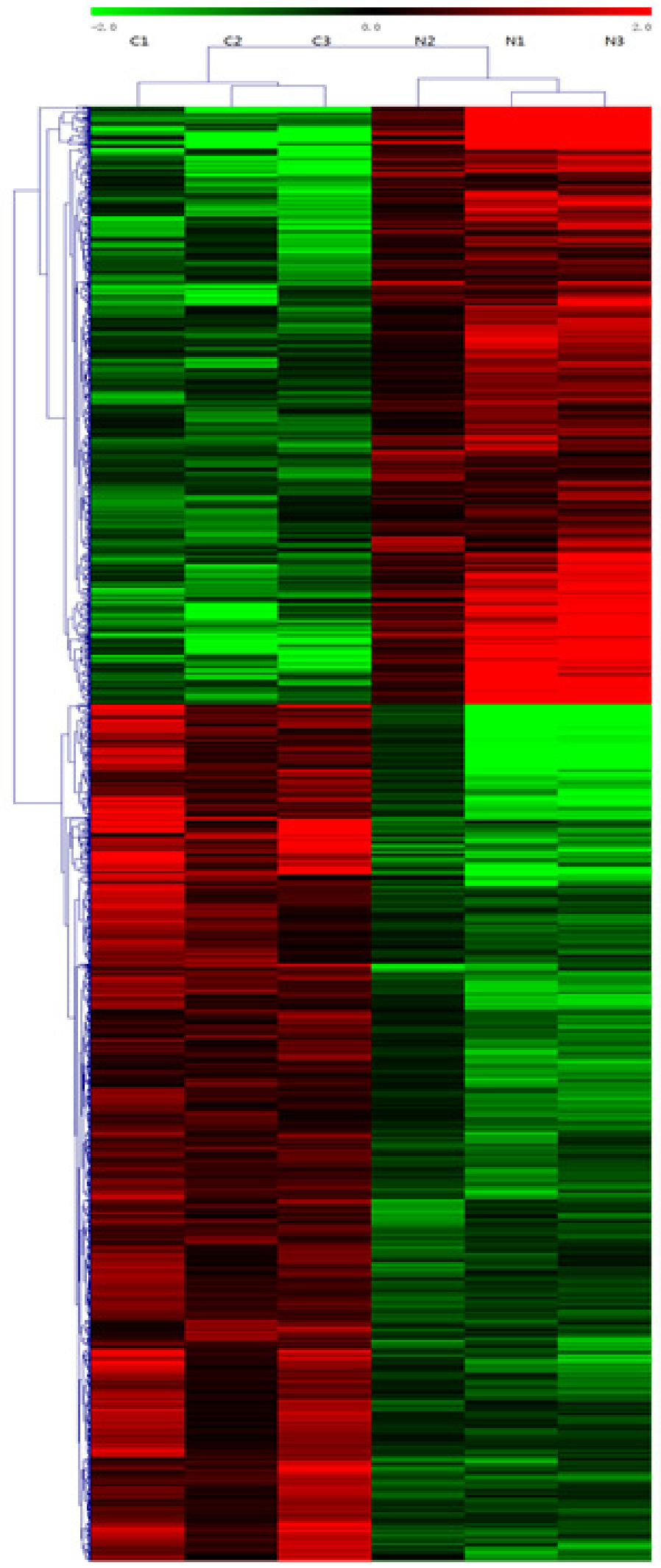

Figure 1: Heat map and hierarchical clustering of IncRNA profile comparison between the TNBC and normal breast samples. Each row represents one lncRNA, and each column represents one tissue sample. The relative lncRNA expression is depicted according to the color scale. Red indicates up-regulation; green indicates down regulation. 2.0, 0 and -2.0 are folds changes in the corresponding spectrum, whereas $\mathrm{N}$ represents normal breast samples tissue and $\mathrm{C}$ represents TNBC tissue. The differentially expressed lncRNAs clearly self-segregated into $\mathrm{N}$ and $\mathrm{C}$ clusters. 
(A).

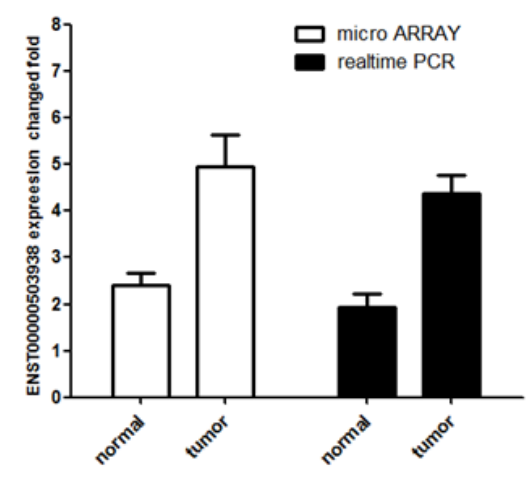

(C).

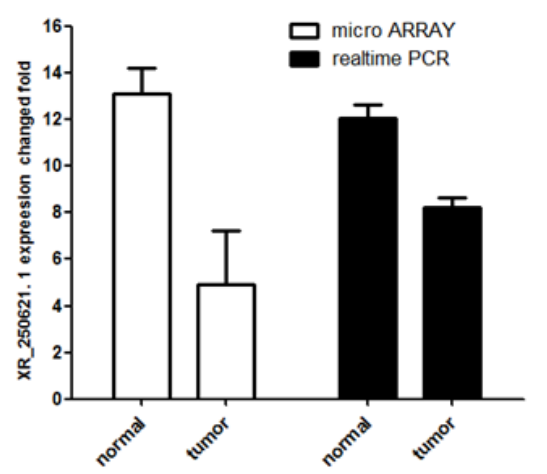

(B).

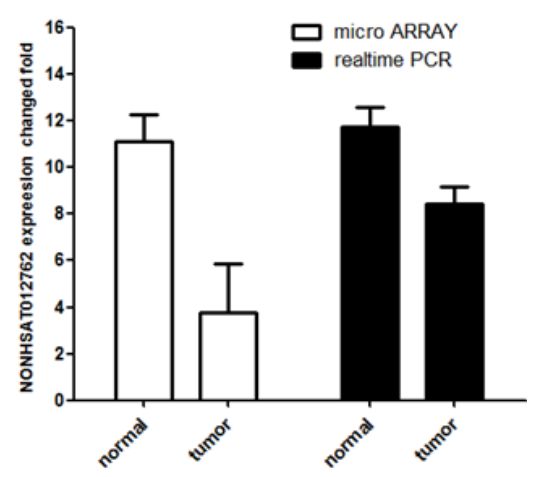

(D)

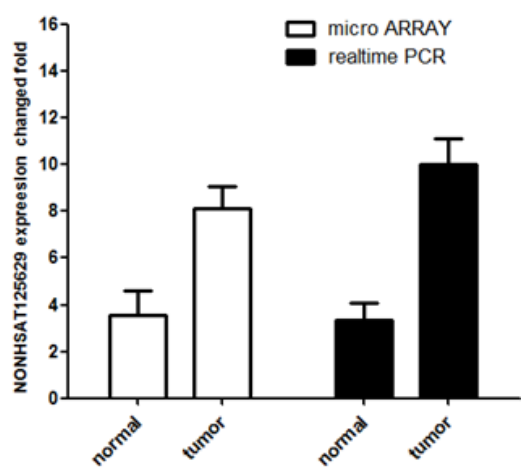

Figure 2: Comparison between microarray data and qPCR results. A. ENST00000503938, B. NONHSAT012762 C. XR_250621.1 and D. NONHSAT125629 which were determined to be differentially expressed in TNBC samples compared with NT samples in 3 paired patients by microarray was validated by qPCR in 12 paired tissues. The heights of the columns in the chart represent the log-transformed median fold changes in expression across the 12 patients for the lncRNA validation; the bars represent standard errors. The validation results of the lncRNAs indicated that the microarray data correlated well with the qPCR results.

(A).

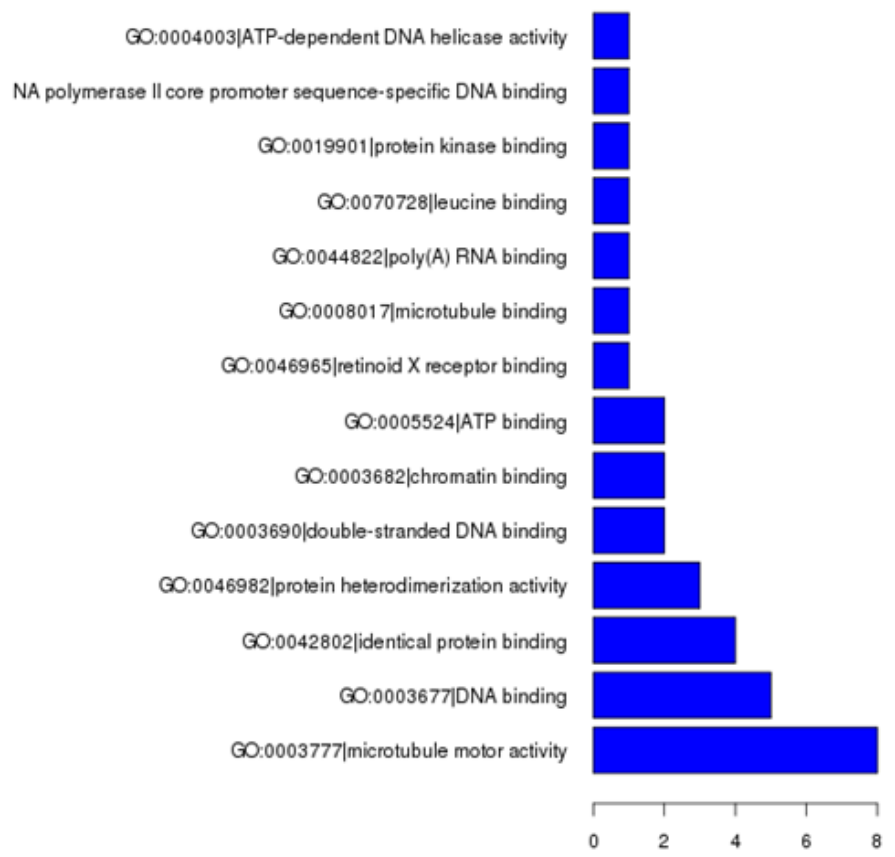

(B).

enrich_stat

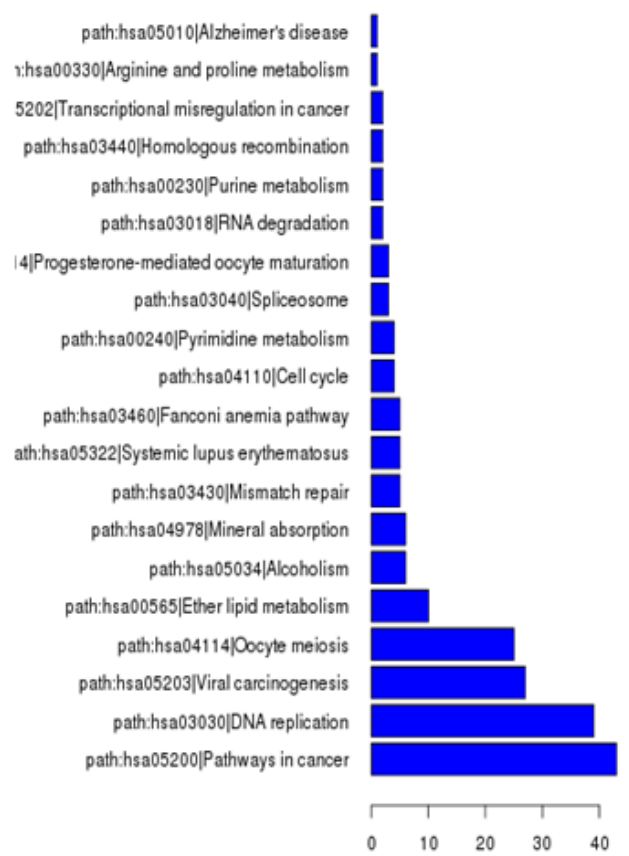

Figure 3: GO analysis A. and KEGG Pathway analysis B. of aberrantly expressed lncRNAs in TNBC. 


\section{Go and KEGG pathway analysis}

To predict the functions of the lncRNAs, we adopted method originally demonstrated in this paper[10]. Briefly, we first calculated the co-expressed mRNAs for each of the differentiated lncRNAs, and then we conducted a functional enrichment analysis of this set of co-expressed mRNAs. The enriched functional terms were used as the predicted functional terms for each given lncRNA. To explore potential biological associations, we ran GO and Pathway analysis with the top 500 differentially expressed lncRNAs and mRNAs. GO analysis indicated that several functional pathways were enriched. Among these pathways, protein binding, fibroblast growth factor-activated receptor activity, structural constituent of ribosome, protein kinase binding and poly(A) RNA binding signaling were the most closely associated with TNBC (Figure 3A). Furthermore, using the same criteria as the GO analysis, KEGG Pathway analysis showed that some pathways corresponded, including ribosome, pathways in cancer, NOD-like receptor signaling pathway, cell cycle, DNA replication, etc. (Figure 3B).

\section{Construction of co-expression network}

To explore which lncRNAs and mRNAs play a critical role in TNBC progression, we constructed a co-expression network based on the correlation analysis between the differentially expressed lncRNAs and mRNAs. LncRNAs and mRNAs with Pearson's correlation coefficients of no less than 0.99 were used to construct the network. To explore lncRNAs that possibly have trans-regulating functions, we compared the mRNAs that coexpressed with these IncRNAs with the mRNAs that are regulatory targets of certain Transcription factors (TFs). Results show that EP300, NFYB and E2F1 may play central roles in lncRNAs process (Figure 4). The coexpression network indicated that one mRNA or IncRNA might correlate with one to ten lncRNAs (Figure 2S). The co-expression network may suggest that the interregulation of lncRNAs and mRNAs is involved in TNBC.

\section{DISCUSSION}

Pathogenesis of breast cancer remains unclear; therefore, further study of breast cancer is of great importance. As lncRNAs constitute an important class of gene expression regulatory factors, their aberrant expression would inevitably lead to abnormal gene expression levels, which might result in tumorigenesis $[2,5]$. To date, there have been few studies studying lncRNAs expression profile in breast cancer or predicating the association of IncRNA expression with clinical pathological features and outcomes in breast cancer [11, $7,12,9,13,14]$. Thus, lncRNAs have opened a new field of breast cancer genomics. Although there are no drugs that act against lncRNAs presently, it will be fascinating to observe whether drugs could be developed that specifically target lncRNAs. Notably, lncRNAs can be detected in human body fluids and hold great promise as biomarkers.

In the present study, we investigated lncRNAs expression signature of TNBC tumor samples from patients. With abundant and varied probes amounting to 78,243 human IncRNAs and 30,215 coding transcripts in the microarray, a large number of lncRNAs could be determined quantitatively and significant differential expression in cancer tissue compared to normal breast tissue was observed. 1,758 lncRNAs and 1,254 mRNAs were found to be significantly differentially expressed. In addition, qRT-PCR was employed to validate the microarray analysis findings, and results were consistent with the data from the microarrays. These results revealed that there were unique lncRNAs expression signatures in these tissues. However, the majority of differentially expressed lncRNAs corresponded to novel transcripts of unknown functions $[4,15]$. In order to obtain insight into lncRNA target gene functions, GO analysis and KEGG pathway annotation were applied to the lncRNA target gene pool. GO analysis revealed that the number of genes corresponding to down-regulated mRNAs were larger than that corresponding to up-regulated mRNAs. These pathways may play important roles in the occurrence and development of TNBC. Increased understanding of the role of these potential endogenous lncRNAs in breast cancer cells could provide additional insight on the role these pathways play in mediating breast cancer progression.

An increasing number of studies have demonstrated that a number of lncRNAs are not transcriptional noise, but have important functions, such as regulating gene expression at various molecular levels, including protein, RNA, miRNA and DNA[16, 17]. Few studies have focused on how lncRNA genes themselves were transcriptionally regulated. Yang et al. developed a system by which users could browse transcription factor binding sites in the regulatory regions of $\operatorname{lncRNAs}$ [18]. However, $\operatorname{lncRNAs}$ are temporally and spatially expressed and regulated, and motif-based sequence analysis cannot capture the dynamic regulation of lncRNAs by transcription factors. In this study, we constructed a transcription factors-lncRNAsmRNA network based on expressions in the TNBC tissue and binding sites in the regulatory regions of a specific lncRNA. Results showed that EP300, NFYB and E2F1 played central roles in lncRNAs process and TNBC development, which were consist with previous reports [19-21]. As more data become available, it will facilitate the research on the transcriptional regulation of lncRNAs.

Several limitations should be acknowledged for this study. First, gene expression microarray have limited dynamic range and lack the ability to discover novel features as splice isoforms or fusion transcripts. RNA-seq 
technology promises to unravel previously inaccessible complexities in the transcriptome, such as allele-specific expression and novel promoters and isoforms. However, datasets produced are large and complex and interpretation is not straight forward. Second, the sample size of each dataset is relatively small, the significance and robustness of the signature requires further confirmation, ideally with large prospective patient cohorts with prognostic date. Last but not least, although the roles of the lncRNAs in TNBC pathogenesis are presently unclear, our findings suggest that lncRNAs deserve further studied. Additional functional investigations of these lncRNAs on cancer cell lines and xenograft models may increase our outstanding of their roles in determining TNBC prognosis.

To summarize, comprehensive in-depth analysis of the expression profiles of lncRNAs was executed in this study. A set of IncRNAs with differential expression were found in TNBC compared with normal breast tissue. Furthermore, potential roles for these lncRNAs in the regulation of protein binding, fibroblast growth

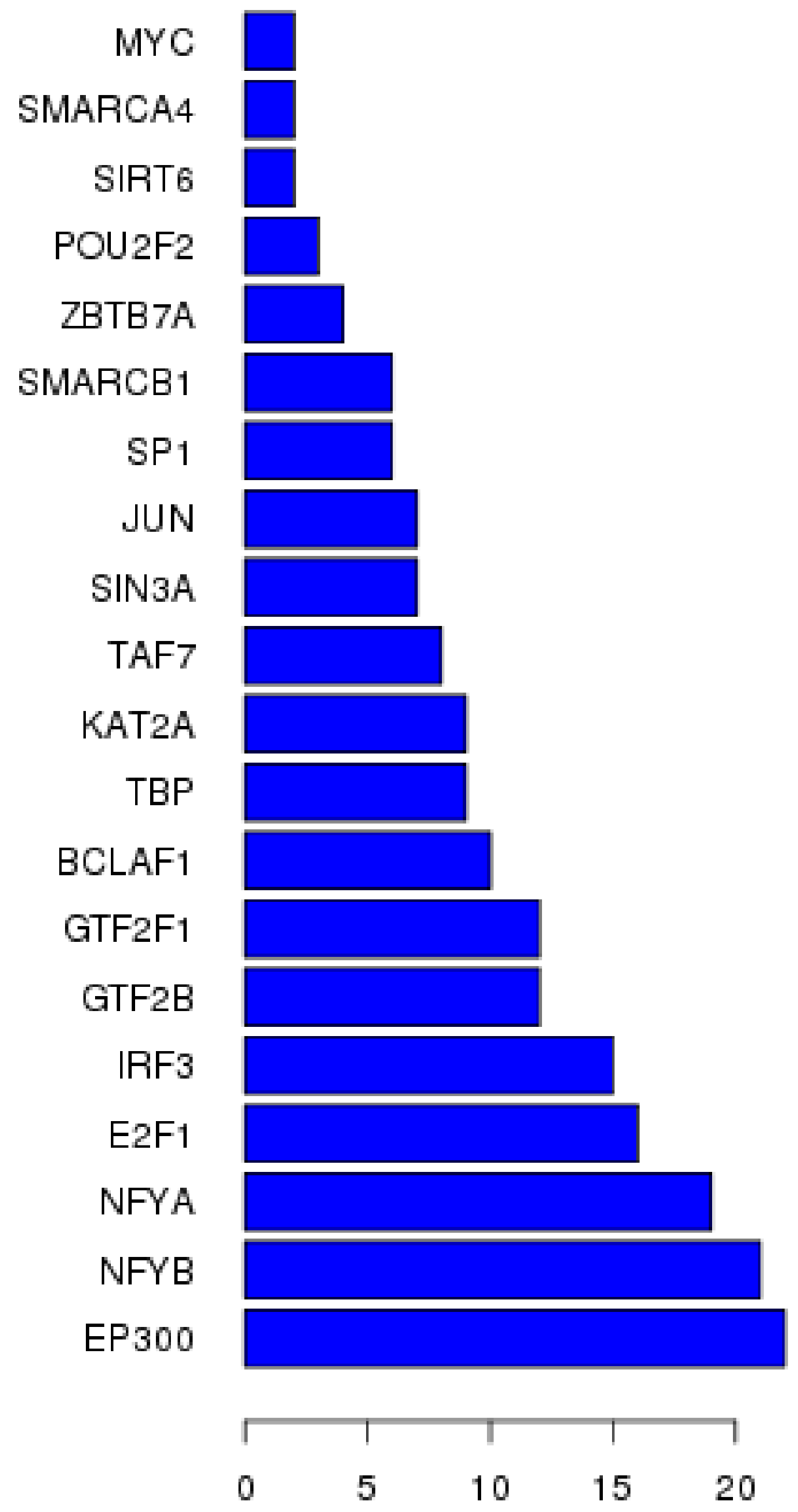

Figure 4: Top transcriptional factors profiling based on aberrantly expressed IncRNAs in TNBC. 
factor-activated receptor activity, structural constituent of ribosome, protein kinase binding and poly (A) RNA binding signaling pathways will be identified. Further investigation of the IncRNAs identified in this study will likely focus on their biological functions and their association with TNBC. Our study provides useful information for exploring potential therapeutic targets for breast cancer.

\section{MATERIALS AND METHODS}

\section{Ethics statement}

This study was approved by the human ethics committee of the Zhejiang Taizhou Hospital, People's Republic of China. All patients are informed and have declared written informed consent that their samples can be used for research.

All patients received tumor resection at Zhejiang Taizhou Hospital and were diagnosed with TNBC histopathologically after surgery. Immunochemical staining of estrogen receptor/progesterone receptor and ErbB2 receptor in 3 samples are shown in Figure S1. There was no radiotherapy or chemotherapy prior to surgery. 3 paired samples were used for microarray analysis of lncRNAs and 12 paired samples were used for an extra evaluation by real-time PCR. Demographic and clinical characterizations of the study population are summarized in Table S1.

\section{Tissue collection and RNA extraction}

Paired TNBC tissues and adjacent normal breast tissues from every subject were snap-frozen in liquid nitrogen immediately after resection and stored at $-80{ }^{\circ} \mathrm{C}$ until use. The mirVana ${ }^{\mathrm{TM}}$ RNA Isolation Kit (Ambion, Foster City, CA, United States) was used to extract total RNA from frozen samples, according to the manufacturer's protocols, which were then eluted with 100 $\mathrm{mL}$ of nuclease-free water. Total RNA was quantified with the NanoDrop ND-2000 (Thermo Scientific) and the RNA integrity was assessed using Agilent Bioanalyzer 2100 (Agilent Technologies).

\section{LncRNA and mRNA microarray expression profiling}

The microarray profiling was conducted in the laboratory of the OE Biotechnology Company in Shanghai, People's Republic of China. The sample labeling, microarray hybridization and washing were performed based on the manufacturer's standard protocols. Briefly, mRNA was purified from total RNA after removal of rRNA by using an mRNA-ONLY Eukaryotic mRNA IsolationKit (Epicentre Biotechnologies, USA). Then, each sample was transcribed to double strand cDNA, then synthesized into cRNA and labeled with Cyanine-3-CTP. The labeled cRNAs were hybridized onto the Human lncRNA array V4.0 $(4 \times 180 \mathrm{~K}$, Agilent $)$, including the global profiling of 78,243 human IncRNAs and 30,215 coding transcripts. After washing, the arrays were scanned with the Agilent Scanner G2505C (Agilent Technologies). Feature Extraction software (version 10.7.1.1, Agilent Technologies) was used to analyze array images and extract the raw data. Genespring (Version 12.5, Agilent Technologies) was employed to finish the basic analysis of the raw data. To begin with, the raw data were normalized with the quantile algorithm. The probes that had at least 1 condition out of 2 conditions flagged as "P" were chosen for further data analysis. Differentially expressed lncRNAs and mRNAs were then identified through fold-change as well as $P$ values calculated with $t$-test. The threshold set for up- and down-regulated genes was fold change $\geq 2.0$ and $p$ value $\leq 0.05$. Afterwards, Hierarchical Clustering was performed to display the distinguishable lncRNAs and mRNAs expression patterns among the samples.

\section{Functional group analysis}

GO analysis and KEGG analysis were applied to determine the biological roles of these differentially expressed mRNAs, base on the latest KEGG (Kyoto Encyclopedia of Genes and Genomes) database (http:// www.genome.jp/kegg/). The $p$ value (Hypergeometric- $P$ value) denotes the significance of the pathway correlated to the conditions. The recommend $p$-value cut-off is 0.05 .

\section{Construction of the co-expression network}

Potentially trans-regulated protein-coding genes were defined as coexpressed and beyond 100 $\mathrm{kb}$ in genomic distance from, or on the other allele of, differentially expressed lncRNAs. The lncRNAsTranscription factors (TFs) network was constructed using hypergeometric cumulative distribution function with the help of MATLAB 2012b (The MathWorks). The graph of the lncRNAs-TFs network was drawn with the help of Cytoscape 3.01 (Agilent and IBS). If the intersection of these two groups is large enough $(P<0.01$, calculated by hypergeometric cumulative distribution function and FDR $<0.01$, under the control of the Benjamini and Hochberg procedure), then we predict that these lncRNAs possibly participate in pathways regulated by these TFs. The recently released ENCODE data on TFs and their regulatory targets were used in our analysis 


\section{Real-time quantitative reverse transcription-PCR}

A two-step reaction process was used for quantification reverse transcription (RT) and PCR. Each RT reaction consisted of $0.5 \mu \mathrm{g}$ RNA, $2 \mu \mathrm{L}$ of Primer Script Buffer, $0.5 \mu \mathrm{L}$ of oligo dT, $0.5 \mu \mathrm{L}$ of random 6 mers and $0.5 \mu \mathrm{L}$ of Primer Script RT Enzyme Mix I (TaKaRa, Japan), in a total volume of $10 \mu \mathrm{L}$. Reactions were performed in a GeneAmp ${ }^{\circledR}$ PCR System 7500 (Applied Biosystems) for $15 \mathrm{~min}$ at $37{ }^{\circ} \mathrm{C}$, followed by heat inactivation of RT for $5 \mathrm{~s}$ at $85^{\circ} \mathrm{C}$. The $10 \mu \mathrm{L}$ RT reaction mix was then diluted 10 -fold in nuclease-free water and held at $-20^{\circ} \mathrm{C}$. At the end of the PCR cycles, melting curve analysis was performed to validate the specific generation of the expected PCR product. All experiments were done in triplicate. The expression levels of lncRNAs were normalized to glyceraldehyde-3-phosphate dehydrogenase and were calculated using the $2-\Delta \Delta \mathrm{Ct}$ method. The primer sequences were designed in the laboratory based on the DNA sequences and is shown in Table S2.

\section{Statistical analysis}

The Statistical Program for Social Sciences (SPSS) 18.0 software (SPSS, Chicago, IL, United States) was employed to perform all the statistical analyses. All data were expressed as the mean $\pm \mathrm{SD}$ or proportions where appropriate. For comparisons, paired $t$-tests and unpaired $t$-tests were performed where appropriate. GraphPad Prism 5.0 for Microsoft Windows (GraphPad Software, San Diego, CA, United States) was used to plot all graphs. $P$ values of 0.05 (two-tailed) were considered statistically significant.

\section{ACKNOWLEDGMENTS}

This work was supported by the National Science Foundation for Distinguished Young Scholars of China (81125025), National Natural Science Foundation of China (81202103, 31370810, 81172240, 81472714, and 81171914) Zhejiang Provincial Natural Science Foundation of China (LY15H160002) and Henan outstanding youth fund projects (2014011016).

\section{CONFLICTS OF INTEREST}

The authors declare no financial conflicts of interest.

\section{Data availability}

The authors confirm that all data underlying the findings are fully available without restriction. All relevant data are within the paper and its Supporting Information files, or in a public repository (submitted to the GEO repository, number is GSE64790).

\section{REFERENCES}

1. Pal SK, Childs BH, Pegram M (2011) Triple negative breast cancer: unmet medical needs. Breast cancer research and treatment 125:627-636. doi:10.1007/s10549-010-1293-1.

2. Hung T, Chang HY (2010) Long noncoding RNA in genome regulation: prospects and mechanisms. RNA biology 7:582-585.

3. Di Gesualdo F, Capaccioli S, Lulli M (2014) A pathophysiological view of the long non-coding RNA world. Oncotarget 5:10976-10996.

4. Zhao W, Luo J, Jiao S (2014) Comprehensive characterization of cancer subtype associated long noncoding RNAs and their clinical implications. Scientific reports 4:6591. doi:10.1038/srep06591.

5. Prensner JR, Chinnaiyan AM (2011) The emergence of lncRNAs in cancer biology. Cancer discovery 1:391-407. doi:10.1158/2159-8290.CD-11-0209.

6. Zhang J, Zhang P, Wang L, Piao HL, Ma L (2014) Long non-coding RNA HOTAIR in carcinogenesis and metastasis. Acta biochimica et biophysica Sinica 46:1-5. doi:10.1093/abbs/gmt117.

7. Vikram R, Ramachandran R, Abdul KS (2014) Functional significance of long non-coding RNAs in breast cancer. Breast cancer 21:515-521. doi:10.1007/s12282-014-0554-y.

8. Tu ZQ, Li RJ, Mei JZ, Li XH (2014) Down-regulation of long non-coding RNA GAS5 is associated with the prognosis of hepatocellular carcinoma. International journal of clinical and experimental pathology 7:4303-4309.

9. Pickard MR, Williams GT (2014) Regulation of apoptosis by long non-coding RNA GAS5 in breast cancer cells: implications for chemotherapy. Breast cancer research and treatment 145:359-370. doi:10.1007/s10549-014-2974-y.

10. Guttman M, Rinn JL (2012) Modular regulatory principles of large non-coding RNAs. Nature 482:339-346. doi:10.1038/nature10887.

11. Chisholm KM, Wan Y, Li R, Montgomery KD, Chang HY, West RB (2012) Detection of long non-coding RNA in archival tissue: correlation with polycomb protein expression in primary and metastatic breast carcinoma. PloS one 7:e47998. doi:10.1371/journal.pone.0047998.

12. Reiche K, Kasack K, Schreiber S, Luders T, Due EU, Naume B, Riis M, Kristensen VN, Horn F, Borresen-Dale AL, Hackermuller J, Baumbusch LO (2014) Long noncoding RNAs differentially expressed between normal versus primary breast tumor tissues disclose converse changes to breast cancer-related protein-coding genes. PloS one 9:e106076. doi:10.1371/journal.pone.0106076.

13. Meng J, Li P, Zhang Q, Yang Z, Fu S (2014) A four-long non-coding RNA signature in predicting breast cancer survival. Journal of experimental \& clinical cancer research : CR 33:84. doi:10.1186/s13046-014-0084-7. 
14. Su X, Malouf GG, Chen Y, Zhang J, Yao H, Valero V, Weinstein JN, Spano JP, Meric-Bernstam F, Khayat D, Esteva FJ (2014) Comprehensive analysis of long noncoding RNAs in human breast cancer clinical subtypes. Oncotarget 5:9864-9876.

15. Gumireddy K, Li A, Yan J, Setoyama T, Johannes GJ, Orom UA, Tchou J, Liu Q, Zhang L, Speicher DW, Calin GA, Huang Q (2013) Identification of a long non-coding RNA-associated RNP complex regulating metastasis at the translational step. The EMBO journal 32:2672-2684. doi:10.1038/emboj.2013.188.

16. Guo L, Zhao Y, Yang S, Zhang H, Wu Q, Chen F (2014) An integrated evolutionary analysis of miRNA-lncRNA in mammals. Molecular biology reports 41:201-207. doi:10.1007/s11033-013-2852-4.

17. Xing Z, Lin A, Li C, Liang K, Wang S, Liu Y, Park PK, Qin L, Wei Y, Hawke DH, Hung MC, Lin C, Yang L (2014) lncRNA Directs Cooperative Epigenetic Regulation Downstream of Chemokine Signals. Cell 159:1110-1125. doi:10.1016/j.cell.2014.10.013.

18. Yang X, Gao L, Guo X, Shi X, Wu H, Song F, Wang B (2014) A network based method for analysis of lncRNAdisease associations and prediction of lncRNAs implicated in diseases. PloS one 9:e87797. doi:10.1371/journal. pone.0087797.

19. Vleugel MM, Shvarts D, van der Wall E, van Diest PJ (2006) p300 and p53 levels determine activation of HIF-1 downstream targets in invasive breast cancer. Human pathology 37:1085-1092. doi:10.1016/j. humpath.2006.03.015.

20. Bryan EJ, Jokubaitis VJ, Chamberlain NL, Baxter SW, Dawson E, Choong DY, Campbell IG (2002) Mutation analysis of EP300 in colon, breast and ovarian carcinomas. International journal of cancer Journal international du cancer 102:137-141. doi:10.1002/ijc.10682.

21. Ngwenya S, Safe S (2003) Cell context-dependent differences in the induction of E2F-1 gene expression by 17 beta-estradiol in MCF-7 and ZR-75 cells. Endocrinology 144:1675-1685. doi:10.1210/en.2002-0009. 